\title{
A COMPARISON PRINCIPLE FOR EQUATIONS OF THE HAMILTON-JACOBI TYPE IN SET-MEMBERSHIP FILTERING*
}

\author{
A. B. KURZHANSKI ${ }^{\dagger}$ AND P. VARAIYA ${ }^{\dagger}$
}

\begin{abstract}
This paper gives comparison principles for first-order PDEs of the Hamilton-JacobiBellman type that arise in the problem of filtering under unknown disturbances with set-membership bounds on the uncertainty. The exact solutions of this problem, given in set-theoretic terms as "information sets," are expressed as level sets to the solutions of some specific types of the HJB equation. But these solutions require complicated calculations. This paper presents an alternative approach that avoids exact solutions in favor of their upper and lower bounds, which in many cases may suffice for solving the required problems. For systems with linear structure ellipsoidal estimates are given, which ensure tight approximations of the convex information sets.
\end{abstract}

Key words: Guaranteed estimation, dynamic programming, set-membership uncertainty, reachability, information set, information state, HJB equation, comparison principle, ellipsoidal calculus.

1. Introduction. The solution to many problems of state estimation and control synthesis for systems described by ODEs may be reduced to the investigation of first order PDEs of the Hamilton-Jacobi-Bellman (HJB) type and their modifications. HJB equations may also be used to calculate forward and backward reachability sets for control systems without disturbances, and the HJBI (HJB-Isaacs) equation may be used for systems with unknown but bounded disturbances $[1,2,3,4,5,6,7]$.

Another application for Hamiltonian techniques is the solution of the "guaranteed" or "set-membership" filtering problem, which is to estimate the state of a dynamic system, based on observations corrupted by unknown and bounded noise with no statistical description. The solution to such a filtering problem is given by "information sets" of states consistent with the system dynamics and the available measurements. In turn, the information sets may be expressed as level sets of certain functions, called "information states," which are the solutions to special types of the HJB equation. The problem of calculating the information sets turns into one of finding reachability sets under state constraints that arrive on-line, in contrast with problems in which state constraints that are given in advance $[8,9,10,11,12]$.

Solutions to equations of the HJB type are rather difficult to calculate, and the design of computational algorithms is still under development [14, 15]. However, for many applied problems one may often be satisfied with approximate solutions that

\footnotetext{
${ }^{*}$ We dedicate this paper to Professor Tyrone E. Duncan on the occasion of his birthday, in recognition of his pioneering contributions to the development of stochastic control theory.

$\dagger$ Electrical Engineering and Computer Science, University of California, Berkeley, CA 94720, USA, E-mail: varaiya@eecs.berkeley.edu
} 
impose a smaller computational burden and may be achieved by substituting the original HJB equations with variational inequalities [16] due to certain comparison principles $([17,18,4])$. This paper provides comparison theorems for HJB equations generated by the guaranteed state estimation (filtering) problem that are applicable to both smooth and non-smooth solutions of the HJB equations. It is also shown that in the case of linear systems with convex constraints on the disturbances this approach may lead to effective external and internal approximations of the information sets using ellipsoidal techniques.

\section{The Set-membership Filtering Problem and the Related HJB Equa-}

tion. Consider the system

$$
\dot{x}=f(t, x, v), t \in\left[t_{0}, \vartheta\right], x\left(t_{0}\right) \in \mathcal{X}^{0},
$$

in which $x \in \mathbb{R}^{n}$ is the state, $v(t) \in \mathcal{Q}(t) \subset \mathbb{R}^{q}$ is the control, and the compact set $\mathcal{X}^{0}$ is the starting set. The function $f(t, x, v)$ is assumed continuous in all variables and satisfies standard conditions of existence, uniqueness and prolongation of solutions within the intervals under consideration; $\mathcal{Q}(t)$ is a set-valued function with convex, compact values, Hausdorff-continuous in time.

Given also is a measurement equation

$$
y(t)=g(t, x)+w(t),
$$

in which $w$ is the measurement 'noise', the unknown disturbance in the measurement channel. We assume $w(t) \in \mathcal{R}(t) \subset \mathbb{R}^{r}$, and the set $\mathcal{R}(t)$ has properties similar to $\mathcal{Q}(t)$. The function $y(t)$ - the available measurement - is assumed to be known.

Problem 2.1. The system (1) is given with measurement equation (2). The starting position $\left\{t_{0}, \mathcal{X}^{0}\right\}$ at time $t_{0}$ and the available measurement $y(t)$ in the interval $t \in\left[t_{0}, \tau\right]$ are also given. One is to specify the information set $\mathcal{X}[\tau]=\mathcal{X}\left(\tau, t_{0}, \mathcal{X}^{0}\right)$ of all states $x(\tau)$ generated by system (1) that are consistent with $y(t)$ of (1) and the constraints $v(t) \in \mathcal{Q}(t), w(t) \in \mathcal{R}^{r}$.

The set $\mathcal{X}[\tau]$ is a guaranteed estimate of the state $x(\tau)$, and includes the unknown actual state $x(\tau)$ of system (1). Hence the on-line state of the overall system that can be computed from the measurements for $t \geq t_{0}$ may be represented by the pair $\{t, \mathcal{X}[t]\}$. (Of course, the on-line state is also trivially represented by the pair $\left.\left\{t, y_{t}(\cdot)\right\}, y_{t}(\cdot)=y(t+\sigma), \sigma \in\left[t_{0}-t, 0\right].\right)$

Given the starting position $\left\{t_{0}, \mathcal{X}^{0}\right\}$ and measurement $y(t), t \in\left[t_{0}, \tau\right]$, it makes sense to construct for system (1), (3) a forward reachability tube $\mathcal{X}[t]=\mathcal{X}\left(t ; t_{0}, \mathcal{X}^{0}\right)$, $t \geq t_{0}$, which emanates from the set-valued position $\left\{t_{0}, \mathcal{X}^{0}\right\}$ and develops under the on-line state constraint [13]

$$
g(t, x) \in y(t)-\mathcal{R}(t) .
$$


Then $\mathcal{X}[t]$ is precisely the information set that solves Problem 2.1 of guaranteed filtering.

The last formulation leads to an alternative problem of dynamic optimization.

Denote

$$
\left.d^{2}(y, \mathcal{R}(t))=\min \{(y-r, y-r)) \mid r \in \mathcal{R}(t)\right\}
$$

Together with (1) consider the equation

$$
\dot{z}(t)=-d^{2}(y(t)-g(t, x(t)), \mathcal{R}(t)), z \in \mathbb{R} .
$$

Lemma 2.1. The information set $\mathcal{X}[\tau]=\mathcal{X}(\tau, \cdot)$ may be expressed as the level set

$$
\mathcal{X}[\tau]=\{x: V(\tau, x, 0) \leq 0\}
$$

of the value function

$$
V(t, x, z)=\min _{v}\left\{d^{2}\left(x\left(t_{0}\right), \mathcal{X}^{0}\right)+\left|z\left(t_{0}\right)\right| \mid x(t)=x, z(t)=z\right\}, t \geq t_{0} .
$$

The minimum is over all functions $v(s) \in \mathcal{Q}(s), s \in\left[t_{0}, t\right]$.

Problem 2.2. The starting position $\left\{t_{0}, \mathcal{X}^{0}\right\}$ and the available measurements $y(t)$, with $t \in\left[t_{0}, \tau\right]$, are given. One is to specify the value function $V(t, x, z)$, with boundary condition

$$
V\left(t_{0}, x, z\right)=d^{2}\left(x, \mathcal{X}^{0}\right)+|z| .
$$

The function $V(t, x, z)$ is henceforward referred to as the information state of system (1), (4). Its 'cross-section' or level set at level 0, namely (5), is in fact the reachability set for this system under the on-line state constraints (3), with $y(t)$ given.

We use a more detailed notation $V(t, x, z)=V\left(t, x, z \mid V\left(t_{0}, \cdot\right)\right)$ for the function $V(t, x, z)$ to emphasize its dependence on the boundary condition $V\left(t_{0}, \cdot\right)$.

LEMMA 2.2. The following property holds:

$$
V\left(t, x, z \mid V\left(t_{0}, \cdot\right)\right)=V\left(t, x, z \mid V\left(s, \cdot \mid V\left(t_{0}, \cdot\right)\right)\right), t_{0} \leq s \leq t
$$

The 'semigroup' property (7) allows one to derive the following equation of HJB type:

$$
V_{t}+\max \left\{\left(V_{x}, f(t, x, v)\right) \mid v \in \mathcal{Q}(t)\right\}-\left(V_{z}, d^{2}(y(t)-g(t, x), \mathcal{R}(t))\right)=0,
$$

with boundary condition

$$
V\left(t_{0}, x, z\right)=d^{2}\left(x, \mathcal{X}^{0}\right)+|z|
$$

Here $V_{t}, V_{x}, V_{z}$ denote the appropriate partial derivatives of $V(t, x, z)$. 
We thus come to the following proposition.

TheOREM 2.1. The information set $\mathcal{X}[\tau]$ is the level set

$$
\mathcal{X}[\tau]=\{x: V(\tau, x, 0) \leq 0\},
$$

in which $V(t, x, z)$ is the solution to equation (8) with boundary condition (9).

In this paper we avoid finding the exact solution for equation (8). Instead we describe approximate solutions using a comparison principle. Then a lower estimate of function $V(t, x, z)$ will have a larger level set that is an external estimate of set $\mathcal{X}[t]$.

3. The HJB equation: lower and upper estimates of solutions. Introduce the notation

$$
\mathcal{H}\left(t, x, z, p, p^{0}\right)=\max \{(p, f(t, x, v)) \mid v \in \mathcal{Q}(t)\}-\left(p^{0}, d^{2}(y(t)-g(t, x), \mathcal{R}(t))\right),
$$

As indicated above (see also $[2,4]$ ), the solution $V(t, x, z)$ of the corresponding "forward" HJB equation

$$
V_{t}+\mathcal{H}\left(t, x, z, V_{x}, V_{z}\right)=0, \quad V\left(t_{0}, x, z\right)=d\left(x, \mathcal{X}^{0}\right)+z, z>0,
$$

allows one to calculate $\mathcal{X}[t]=\mathcal{X}\left(t, t_{0}, \mathcal{X}^{0}\right)$ as the level set

$$
\mathcal{X}[\tau]=\{x: V(\tau, x, 0) \leq 0\} .
$$

Here $d(x, \mathcal{X})=\inf \left\{(x-m, x-m)^{1 / 2} \mid m \in \mathcal{X}\right\}$ is the Euclidean distance from $x$ to the set $\mathcal{X}$. Property (11) is independent of whether $V$ is a classical or a generalized solution of equation (10).

Thus the exact description of set $\mathcal{X}[\tau]$ requires one to solve the first-order PDE (10). This PDE is often difficult to solve because the reachability sets for nonlinear systems can have a complicated form.

We therefore develop comparison theorems that provide lower and upper estimates of $V(t, x)$. Their level sets yield external and internal estimates of sets $\mathcal{X}[\tau]$.

Assumption 3.1. Given are functions $H\left(t, x, z, p, p^{0}\right), w^{+}(t, x, z) \in C_{1}$ and $\mu(t) \in L_{1}$, satisfying the inequalities

$$
\begin{gathered}
\mathcal{H}\left(t, x, z, p, p^{0}\right) \leq H\left(t, x, z, p, p^{0}\right), \quad \forall\left\{t, x, z, p, p^{0}\right\}, \\
w_{t}^{+}+H\left(t, x, z, w_{x}, w_{z}\right) \leq \mu(t) .
\end{gathered}
$$

Theorem 3.1. Suppose $H\left(t, x, z, p, p^{0}\right), w^{+}(t, x, z), \mu(t)$ satisfy Assumption 3.1. Then the following estimate for the information set $\mathcal{X}[t]$ holds:

$$
\mathcal{X}[t] \subseteq \mathcal{X}_{+}[t]
$$


with

$$
\mathcal{X}_{+}[t]=\left\{x: w^{+}(t, x, 0) \leq \int_{t_{0}}^{t} \mu(s) d s+\max \left\{w^{+}\left(t_{0}, x, 0\right) \mid x \in \mathcal{X}^{0}\right\}\right\}
$$

Consider a pair $x^{*} \in \mathcal{X}[t], v^{*}(s) \in \mathcal{Q}(s), \quad s \in\left[t_{0}, t\right]$, so that the corresponding trajectory $x^{*}\left(s, t, x^{*} \mid v^{*}(\cdot)\right)=x^{*}[s] \in \mathcal{X}[s]$. Then

$$
\begin{gathered}
d w^{+}(t, x, z) /\left.d t\right|_{x=x^{*}(t)}= \\
=w_{t}^{+}\left(t, x^{*}, z\right)+\left(w_{x}^{+}\left(t, x^{*}, z\right), f\left(t, x^{*}, v^{*}\right)\right)-w_{z}^{+}\left(t, x^{*}, z\right) d^{2}\left(y(t)-g\left(t, x^{*}(t)\right), \mathcal{R}(t)\right) \leq \\
\leq w_{t}^{+}\left(t, x^{*}, z\right)+\mathcal{H}\left(t, x^{*}, z, w_{x}^{+}, w_{z}^{+}\right) \leq w_{t}^{+}\left(t, x^{*}, z\right)+H\left(t, x^{*}, w_{x}^{+}, 1\right) \leq \mu(t),
\end{gathered}
$$

and hence

$$
d w^{+}(s, x, z) /\left.d s\right|_{x=x^{*}(s), z(s)=0} \leq \mu(s) .
$$

Integrating this inequality from $t_{0}$ to $t$ gives

$$
w^{+}(t, x, 0) \leq \int_{t_{0}}^{t} \mu(s) d s+w\left(t_{0}, x\left(t_{0}\right), 0\right) \leq \int_{t_{0}}^{t} \mu(s) d s+\max \left\{w^{+}\left(t_{0}, x, 0\right) \mid x \in \mathcal{X}^{0}\right\},
$$

which implies $x^{*}(t) \in \mathcal{X}_{+}[t]$ and the theorem is proved.

Recall that when function $V(t, x)$ is not differentiable, equation (12) is an expression in a formal symbolic notation whose solution should be considered in a "viscosity" sense ( [19], [20], [21]) or equivalent "minimax" sense ([22]).

We move to the discussion of internal estimates for the information sets $\mathcal{X}[t]=$ $\mathcal{X}\left(t, t_{0}, \mathcal{X}^{0}\right)$ and the related HJB equations. As in the above, we do not necessarily require differentiability of the value function $V(t, x, z)$.

Consider the next assumption.

Assumption 3.2. Given are functions $H_{-}\left(t, x, z, p, p^{0}\right)$ and $w^{-}(t, x, z) \in C_{1}$, satisfying the inequalities

$$
\begin{gathered}
\text { (i) } \quad \mathcal{H}\left(t, x, z, p, p^{0}\right) \geq H_{-}\left(t, x, z, p, p^{0}\right), \forall\left\{t, x, z, p, p^{0}\right\}, \\
\text { (ii) } w_{t}^{-}(t, x)+H_{-}\left(t, x, z, w_{x}^{-}(t, x, z), w_{z}^{-}(t, x, z)\right) \geq 0 \\
(\text { iii }) \quad w^{-}\left(t_{0}, x, z\right) \geq V\left(t_{0}, x, z\right), z \geq 0 .
\end{gathered}
$$

Under Assumption 3.2(i,ii) we have

$$
w_{t}^{-}+\mathcal{H}\left(t, x, w_{x}^{-}, w_{z}^{-}\right) \geq w_{t}^{-}+H_{-}\left(t, x, w_{x}^{-}, w_{z}^{-}\right) \geq 0 .
$$


Integrating the last inequality from $\vartheta$ to $t_{0}$ along a trajectory $x[t] \in \mathcal{X}[t], t \in\left[t_{0}, \vartheta\right]$, with $x[\vartheta]=x$, gives

$$
w^{-}(\vartheta, x, 0)-w^{-}\left(t_{0}, x\left[t_{0}\right], 0\right)+V\left(t_{0}, x\left[t_{0}\right], 0\right)-V(\vartheta, x, 0) \geq 0
$$

and so, under Assumption 3.2 (iii),

$$
w^{-}(\vartheta, x, 0) \geq w^{-}\left(t_{0}, x\left[t_{0}\right], 0\right) \geq V\left(t_{0}, x\left[t_{0}\right], 0\right)=V(\vartheta, x, 0)
$$

which brings us to the next proposition.

THEOREM 3.2. Under Assumption 3.2 the following inequality is satisfied:

$$
V(\vartheta, x, 0) \leq w_{-}(\vartheta, x, 0)
$$

This proposition is similar to those of 7.5-7.7 from [17].

Denoting $\mathcal{X}^{-}[t]=\left\{x: w^{-}(t, x, 0) \leq 0\right\}, t \geq t_{0}$ and using the last inequality, we reach the conclusion.

COROLlary 3.1. Under Assumption 3.2 the following inclusion holds:

$$
\mathcal{X}_{-}[t] \subseteq \mathcal{X}[t]
$$

The specificity of the estimation problem considered here is that the measurement $y(t)$ arrives on-line. Therefore, the class of functions $y(t)$ under consideration will depend on the class of functions $w(t)$ that generate the measurement noise. In particular, $y(t)$ may be taken continuous if the functions $w(t)$ are, too.

REMARK 3.1. Since the information set $\mathcal{X}[\tau]$ is the level set of the information state (the function $V(\tau, x, z)$ ), the current position of the system may be defined not only as one of the pairs $\{\tau, \mathcal{X}[\tau]\},\left\{\tau, y_{\tau}(\cdot)=y(t+\sigma), \sigma \in\left[t_{0}-t, 0\right]\right\}$, as indicated before, but also as the pair $\{\tau, V(\tau, x, z)\}$.

Taking $\{\tau, V(\tau, x, z)\}$ to be the position appears more convenient, because its evolution may be more easily expressed through conventional HJB partial differential equations, rather than through less familiar constructions such as funnel equations and the like required for $\{\tau, \mathcal{X}[\tau]\}$.

We now pass to the class of linear systems with convex constraints for which the results of the previous sections may be developed with greater detail.

4. The Linear-Convex Case . Given the linear system

$$
\dot{x}=A(t) x+B(t) u+C(t) v
$$

in which $u(t) \in \mathcal{R}^{p}$ is the given control and $v(t) \in \mathcal{R}^{q}$ is the unknown disturbance. Given also is a measurement equation

$$
y(t)=G(t) x+w(t),
$$


in which $y(t)$ is the available measurement and $w(t) \in \mathcal{R}^{r}$ is the unknown disturbance in the measurement channel. The disturbances $v, w$ are taken to be bounded with known bounds given by nondegenerate ellipsoids,

$$
v \in \mathcal{Q}(t)=\mathcal{E}(q, Q(t)) ; \quad w \in \mathcal{R}(t)=\mathcal{E}(r(t), \mathcal{R}(t))
$$

with $Q^{\prime}(t)=Q(t)>0, R^{\prime}(t)=R(t)>0$. The ellipsoid

$$
\mathcal{E}(q, Q)=\left\{v:\left(v-q, Q^{-1}(v-q)\right) \leq 1\right\},
$$

has support function

$$
\rho(l \mid \mathcal{E}(q, Q))=\max \{(l, q) \mid q \in \mathcal{E}(q, Q)\}=(l, q)+(q, P, q)^{1 / 2} .
$$

Given also is an ellipsoidal bound on the initial vector $x\left(t_{0}\right)$,

$$
x\left(t_{0}\right) \in \mathcal{X}^{0}=\mathcal{E}\left(x^{0}, X^{0}\right),
$$

so that the starting position is $\left\{t_{0}, \mathcal{E}\left(x^{0}, X^{0}\right)\right\}$.

These relations will be referred to as describing the linear-convex case with ellipsoidal bounds. In this special case of Problem 2.1 we assume that the control $u(t)$ is known. Note that for given $y(t)$ equation (18) imposes an on-line state constraint

$$
G(t) x(t) \in y(t)-r(t)+\mathcal{E}(0, R(t))=\mathcal{R}_{y}(t) .
$$

For this case we now specify the alternative problem of dynamic optimization stated in Section 2. Let

$$
\begin{gathered}
d^{2}(y, \mathcal{E}(r, R))=\max \{0,(y-r, R(y-r))-1\}, \\
k^{2}(t)=(y(t)-G(t) x-r(t), R(t)(y(t)-G(t) x-r(t))) .
\end{gathered}
$$

Together with (17) consider the equation

$$
\dot{z}=-d^{2}(y(t)-G(t) x, \mathcal{E}(r(t), R(t))), z \in \mathbb{R}, z \geq 0 .
$$

Lemma 4.1. The information set $\mathcal{X}[\tau]$ may be expressed as the level set

$$
\mathcal{X}[\tau]=\{x: V(\tau, x, 0) \leq 0\}
$$

for the value function

$$
V(t, x, z)=\min _{u}\left\{d^{2}\left(x\left(t_{0}\right), \mathcal{E}\left(x^{0}, X^{0}\right)\right)+\left|z\left(t_{0}\right)\right| \mid x(t)=x, z(t)=z\right\}, t \geq t_{0} .
$$

The HJB equation for $V(t, x, z)$ is

$$
V_{t}+\left(V_{x}, A(t) x+B(t) u(t)+C(t) q(t)\right)+
$$




$$
\max \left\{\left(V_{x}, C(t) v\right) \mid v \in \mathcal{E}(0, Q(t))\right\}-\left(V_{z}, d^{2}(y(t)-G(t) x, \mathcal{E}(r(t), R(t)))=0,\right.
$$

with boundary condition

$$
V\left(t_{0}, x, z\right)=d^{2}\left(x, \mathcal{E}\left(x^{0}, X^{0}\right)\right)+|z| .
$$

As before $V_{t}, V_{x}, V_{z}$ are the partial derivatives of $V(t, x, z)$.

In greater detail, with $z \geq 0, t \geq t_{0}$, equation (23) turns into

$$
\begin{gathered}
V_{t}+\left(V_{x}, A(t) x+B(t) u(t)+C(t) q(t)\right)+ \\
\left(V_{x}, C(t) Q(t) C^{\prime}(t) V_{x}\right)^{1 / 2}-\left(V_{z}, \mathcal{I}(h(t, x)>0) h(t, x)\right)=0,
\end{gathered}
$$

with $h(t, x)=k^{2}(t, x)-1$. Here $\mathcal{I}(h>0)=1$, if $h>0$ and $\mathcal{I}(h>0)=0$ if $h \leq 0$, so that $\mathcal{I}(h>0)$ is the indicator function for the semiaxis $h>0$.

We thus come to the following proposition.

TheOREM 4.1. The information set $\mathcal{X}[\tau]$ is the level set

$$
\mathcal{X}[\tau]=\{x: V(\tau, x, 0) \leq 0\},
$$

in which $V(t, x, z)$ is the solution to equation (25) with boundary condition (24).

We now specialize the results of Section 3 for linear convex systems with ellipsoidal bounds. We shall approximate the information state $V(t, x, z)$ by parameterized quadratic forms. The corresponding information sets $\mathcal{X}[t]$ will then be approximated by parameterized families of ellipsoids, [23], [24].

5. Ellipsoidal Estimates. We shall now approximate the solution $\mathcal{X}[t]$ of equation (17), (19), $x\left(t_{0}\right) \in \mathcal{X}[t]$ by external ellipsoids.

Denote

$$
\begin{gathered}
\mathcal{H}\left(t, x, z, p, p^{0}\right)=(p, A(t) x+B(t) u(t)+C(t) q(t))+\left(p, C(t) Q(t) C^{\prime}(t) p\right)^{1 / 2}- \\
-\left(p^{0}, \mathcal{I}(h(t, x)>0) h(t, x)\right)
\end{gathered}
$$

and recall the relation

$$
\left(p, C(t) Q(t) C^{\prime}(t) p\right)^{1 / 2} \leq \gamma^{2}(t)+\left(4 \gamma^{2}\right)^{-1}\left(p, C(t) Q(t) C^{\prime}(t) p\right), \quad \forall p \in \mathbb{R}^{n},
$$

with equality reached for $\gamma^{2}(t)=(1 / 2)\left(p, C(t) Q(t) C^{\prime}(t)\right)^{1 / 2}$.

Denoting

$$
\begin{gathered}
H\left(t, x, z, p, p^{0}\right)=(p, A(t) x+B(t) u(t)+C(t) q(t))+\gamma^{2}(t)+\left(4 \gamma^{2}\right)^{-1}\left(p, C(t) Q(t) C^{\prime}(t) p\right)- \\
-\left(p^{0}, \mathcal{I}(h(t, x)>0) h(t, x)\right),
\end{gathered}
$$


we observe that $\mathcal{H}\left(t, x, z, p, p^{0}\right) \leq H\left(t, x, z, p, p^{0}\right)$ for all values of the variables $\{t, x, z$, $\left.p, p^{0}\right\}$.

Let us now approximate the function $V\left(t, x, z, p, p^{0}\right)$ in the domain $x \in \mathbb{R}^{n}, z \geq$ $0, t \geq t_{0}$ by a quadratic function of type

$$
w(t, x, z)=\left(x-x^{*}(t), K(t)\left(x-x^{*}(t)\right)+z-1,\right.
$$

with differentiable $K(t)=K^{\prime}(t)>0$.

Let us first assume $k^{2}(t, x)-1>0$, so that $\mathcal{I}(h(t, x)>0)=1$. Then we have

$$
\begin{aligned}
& w_{t}+\mathcal{H}\left(t, x, z, w_{x}, w_{z}\right) \\
= & w_{t}+\left(w_{x}, A(t) x+B(t) u(t)+C(t) q(t)\right) \\
& \left.+\left(w_{x}, C(t) Q(t) C^{\prime}(t) w_{x}\right)^{1 / 2}-\mathcal{I}\left(k^{2}(t, x)-1>0\right)\left(k^{2}(t, x)-1\right)\right) \\
\leq & w_{t}+H\left(t, x, z, w_{x}, w_{z}\right) \\
\leq & w_{t}+\left(w_{x}, A(t) x+B(t) u(t)+C(t) q(t)\right) \\
& +\gamma^{2}(t)+\left(4 \gamma^{2}(t)\right)^{-1}\left(\left(w_{x}, C(t) Q(t) C^{\prime}(t) w_{x}\right)\right. \\
& -(y(t)-G(t) x-r(t), R(t)(y(t)-G(t) x-r(t))+1 .
\end{aligned}
$$

Substituting $w(t, x, z)=\left(x-x^{*}(t), K(t)\left(x-x^{*}(t)\right)+z\right.$, we obtain

$$
\begin{aligned}
& w_{t}+\mathcal{H}\left(t, x, z, w_{x}, w_{z}\right) \\
\leq & \left(x-x^{*}(t), \dot{K}(t)\left(x-x^{*}(t)\right)\right)+2\left(K\left(x-x^{*}(t)\right), A(t) x+B(t) u(t)+C(t) q(t)\right) \\
& +\left(\gamma^{2}(t)\right)^{-1}\left(K\left(x-x^{*}(t), C(t) Q(t) C^{\prime}(t) K\left(x-x^{*}(t)\right)\right)+\gamma^{2}(t)\right. \\
& -\alpha((y(t)-G(t) x-r(t), R(t)(y(t)-G(t) x-r(t)))-1),
\end{aligned}
$$

with $\alpha=1$.

In case $k^{2}(t, x)-1 \leq 0$ we will have the same inequality (27), except that now $\alpha=0$.

Continuing further with $\alpha=1$, we demand that the next equality holds:

$$
\begin{gathered}
\left(\left(x-x^{*}(t), \dot{K}(t)\left(x-x^{*}(t)\right)-2\left(\dot{x}^{*}(t), K(t)\left(x-x^{*}(t)\right)+\right.\right.\right. \\
+2\left(K(t)\left(x-x^{*}(t), A(t)\left(x-x^{*}(t)\right)+A(t) x^{*}(t)+B(t) u(t)+C(t) q(t)\right)+\right. \\
+\left(\gamma^{2}(t)\right)^{-1}\left(\left(K(t)\left(x-x^{*}(t)\right), C(t) Q(t) C^{\prime}(t) K(t)\left(x-x^{*}(t)\right)+\gamma^{2}-\right.\right. \\
\alpha\left(G(t)\left(x-x^{*}(t)\right), R(t) G(t)\left(x-x^{*}(t)\right)\right)+\left(G(t) x^{*}(t), R(t) G(t) x^{*}(t)\right)+ \\
+2\left(G(t) x^{*}(t), R(t) G(t)\left(x-x^{*}(t)\right)\right)-2\left(y(t)-r(t), R(t) G(t)\left(x-x^{*}(t)\right)-\right.
\end{gathered}
$$




$$
-2\left(y(t)-r(t), R(t) G(t) x^{*}(t)\right)+(y(t)-r(t), R(t)(y(t)-r(t)))=0 .
$$

Equating to zero the terms with multipliers of second order in $x-x^{*}$, then those of first order in the same variable, we observe that the last equality will be fulfilled if and only if the following equations hold:

$$
\begin{gathered}
\dot{K}+K^{\prime} A(t)+A^{\prime}(t) K+\left(\gamma^{2}(t)\right)^{-1} K C(t) Q(t) C^{\prime}(t) K-\alpha G^{\prime}(t) R(t) G(t)=0, \\
\dot{x}^{*}=A(t) x^{*}(t)+B(t) u(t)+C(t) q(t)+\alpha G^{\prime}(t) R(t)\left(y(t)-G(t) x^{*}-r(t)\right),
\end{gathered}
$$

with boundary conditions

$$
K\left(t_{0}\right)=\left(X^{0}\right)^{-1}, x^{*}\left(t_{0}\right)=x^{0} .
$$

Conditions (27)-(29) together with some further calculation yield the inequality

$$
d w / d t \leq \gamma^{2}(t)+\alpha,(\alpha=1),
$$

which follows from (27) if $h(t, x)>0$. If $h(t, x) \leq 0$, relation (30) holds for $\alpha=0$.

We therefore have (30) where $\alpha$ is either one or zero.

Now recall that $w(t, x, z)=\left(x-x^{*}(t), K(t)\left(x-x^{*}(t)\right)+z-1\right.$, and since we chose $K\left(t_{0}\right)=\left(X^{0}\right)^{-1}$ we have also satisfied Assumption 3.2(iii). Hence we have $w\left(t_{0}, x, z\right) \leq V\left(t_{0}, x, z\right)$.

Let us now integrate the last inequality along a trajectory $x[t]=x(t ; \vartheta, x), z[t]=$ $z(t ; \vartheta, z)$ with $x(\vartheta)=x, z(\vartheta)=z$, that runs within the exact information set $\mathcal{X}[t]$ (which happens under $x \in \mathcal{X}[\vartheta], z=0$ and disturbances $v(t) \in \mathcal{E}(q(t), Q(t)), w(t) \in$ $\mathcal{E}(r(t), R(t)))$. Then we have

$$
\begin{aligned}
& w(\vartheta, x, 0)-\int_{t_{0}}^{\vartheta} \gamma^{2}(s) d s \\
\leq & w\left(t_{0}, x\left(t_{0}\right), 0\right)-\int_{t_{0}}^{\vartheta} \mathcal{I}(h(t, x[s])>0) h\left(t, x^{*}[s]\right) d s=w\left(t_{0}, x\left(t_{0}\right), 0\right) \\
\leq & V\left(t_{0}, x\left[t_{0}\right], 0\right)=V(\vartheta, x, 0),
\end{aligned}
$$

so that

$$
\mathbf{w}(\vartheta, x, 0)=w(\vartheta, x, 0)-\int_{t_{0}}^{\vartheta} \gamma^{2}(s) d s \leq V(\vartheta, x, 0) .
$$

In the general case $(z>0)$ we have

$$
\begin{gathered}
w(\vartheta, x, z)-\int_{t_{0}}^{\vartheta}\left(\gamma^{2}(s)+\alpha(s)\right) d s \leq \\
\leq w\left(t_{0}, x\left[t_{0}\right], z\left[t_{0}\right]\right) \leq V\left(t_{0}, x\left[t_{0}\right], z\right)=V(\vartheta, x, z),
\end{gathered}
$$


Here $\alpha(t)=\alpha(z[t])=1$ if $z[t]>0$ and $\alpha(t)=\alpha(z[t])=0$ if $z[t]=0$.

THEOREM 5.1. The lower estimate for $V(\vartheta, x, 0)$ is given according to (32) by function $\mathbf{w}(t, x, 0)$, where $K(t), x^{*}(t)$ are given by (28), (29).

We further transform equation (28) to new variables, substituting variable $K$ by $\mathcal{K}_{+}$according to relations

$$
\mathcal{K}_{+}=K^{-1}, \quad \dot{\mathcal{K}}_{+}=-\mathcal{K}_{+} \dot{K} \mathcal{K}_{+}, \mathcal{K}_{+}(t)=\left(1+\int_{t}^{\vartheta}\left(\gamma^{2}(s)+\alpha(s)\right) d s\right) \mathcal{K}(t)
$$

We then come to equations

$$
\dot{\mathcal{K}}_{+}=A(t) \mathcal{K}_{+}+\mathcal{K}_{+} A^{\prime}(t)+(\pi(t)+\beta(t)) \mathcal{K}_{+}-(\pi(t))^{-1} C(t) Q(t) C^{\prime}(t)-
$$

$$
-\beta(t)\left(\mathcal{K}_{+} G^{\prime}(t) R(t) G(t) \mathcal{K}_{+}\right)
$$

and boundary condition $\mathcal{K}_{+}\left(t_{0}\right)=X^{0}$.

Here

$$
\pi(t)=\gamma^{2}(t) /\left(1+\int_{t}^{\vartheta}\left(\gamma^{2}(s)+\alpha(s)\right) d s\right), \quad \beta(t)=\alpha(t) /\left(1+\int_{t}^{\vartheta}\left(\gamma^{2}(s)+\alpha(s)\right) d s\right)
$$

where $\gamma^{2}(t) \geq 0$ and $\alpha(t)$ is either one or zero.

A particular difficulty of equations (28), (29), (34) is that they contain the multiplier $\alpha(t)=\alpha(z[t])$, which is either 1 or zero, depending on $z[t]$ that depends on $x[t]$, which is unknown - in fact it is being estimated. This difficulty may be overcome as follows.

The measurement $y(t)$ is said to be informative if it brings an "innovation" for all $t \geq t_{0}$, so that the state constraint is always "active." That is, if the starting set $\mathcal{X}^{0}$, the measurement $y(t)$ and the constraints on $v(t), w(t)$ are such that in the reachability tube of system (1), (17) without state constraints, there always exists a trajectory violating the state constraint (3), (19) throughout the whole time interval under consideration, then the measurement $y(t)$ is informative. In this case we may take $\alpha(t) \equiv 1, \forall t \geq t_{0}$.

The formulas derived here then allow the next conclusion.

TheOREM 5.2. Suppose the measurement $y(t)$ is informative. Then the following inclusion (external estimate) holds:

$$
\mathcal{X}[t] \subseteq \mathcal{X}_{+}[t]
$$

in which

$$
\mathcal{X}_{+}[t]=\left\{x:\left(x-x^{*}(t), \mathcal{K}_{+}^{-1}(t)\left(x-x^{*}(t)\right)\right) \leq 1\right\},
$$

whatever be the functions $\pi(t), \beta(t)$ of respective classes. 
REMARK 5.1. The last theorem indicates that the exact set $\mathcal{X}[t]$ lies within the intersection of sets $\mathcal{X}_{+}[t]$ over all functions $\pi(t), \beta(t)$ in their respective classes.

Let $\mathcal{G}(s, t, \mathcal{X}[t]), t \geq t_{0}, s \geq t$, be the reach set of system (17) without state constraint (19), starting from information set $\mathcal{X}[t]$. Denote $\mathcal{F}(s, t)=G(s) \mathcal{G}(s, t, \mathcal{X}[t])$ The next proposition is true.

Lemma 5.1. Suppose

$$
\max _{l}\left\{\rho(l \mid \mathcal{F}(s, t))-\rho\left(l \mid \mathcal{R}_{y}(s)\right) \mid(l, l)=1\right\}>0, \forall s \in(t, t+\sigma], \sigma>0, \forall t \geq t_{0} .
$$

Then the measurement $y(t)$ is informative, so that one may take $\alpha(t) \equiv 1, t \geq t_{0}$.

REMARK 5.2. If $\mathcal{X}^{0} \supset \mathcal{R}_{y}\left(t_{0}\right)$, then one may find the first point $\tau^{*}$ of intersection of $\mathcal{F}\left(s, t_{0}\right)$ with $\mathcal{R}_{y}(s)$ and presume that from $\tau^{*}$ onward the last lemma is true.

The time $\tau^{*}$ may be calculated as follows. Suppose the support function

$$
\rho\left(l \mid \mathcal{F}\left(t_{0}, t_{0}\right)\right)<\rho\left(l \mid \mathcal{R}_{y}\left(t_{0}\right)\right), \forall l .
$$

Then $\tau^{*}$ will be the smallest root of equation $\sigma(s)=0$,

$$
\sigma(s)=\min _{l}\left\{\rho\left(l \mid \mathcal{R}_{y}(s)\right)-\rho(l \mid \mathcal{F}(s)) \mid(l, l)=1\right\} .
$$

(Clearly, $\sigma\left(t_{0}\right)>0$, so that $\left.\tau^{*}>t_{0}\right)$.

We now pass to the calculation of internal ellipsoidal estimates for $\mathcal{X}[t]$. Starting from relation (26) and applying inequality

$$
\left(p, C(t) Q(t) C^{\prime}(t) p\right)^{1 / 2} \geq(T(t) p, T(t) p)^{-1 / 2}\left(T(t) p, S(t)\left(C(t) Q(t) C^{\prime}(t)\right)^{1 / 2} p\right)
$$

where matrices $T(t)=T^{\prime}(t)$ are symmetric and matrices $S(t)$ are orthogonal $(S(t)$ $\left.S^{\prime}(t)=I\right)$, we come to

$$
\mathcal{H}\left(t, x, z, p, p^{0}\right) \geq H_{-}\left(t, x, z, p, p^{0}\right)
$$

with

$$
\begin{aligned}
H_{-}\left(t, x, z, p, p^{0}\right)= & (p, A(t) x+B(t) u(t)+C(t) q(t)) \\
& -(T(t) p, T(t) p)^{-1 / 2}(T(t) p, S(t) \mathbf{C}(t) p)-\left(p^{0}, \mathcal{I}(h(t, x)>0) h(t, x)\right),
\end{aligned}
$$

and $\mathbf{C}(t)=\left(C(t) Q(t) C^{\prime}(t)\right)^{1 / 2}$.

Selecting $w_{-}(t, x, z)=\left(x-x^{*}(t), \mathcal{K}_{-}^{-1}(t)\left(x-x^{*}(t)\right)+|z|-1\right.$ with $T(t)=\mathcal{K}_{-}(t)$ and estimating the total derivative $d w_{-}(t, x, z) / d t$ along the scheme above, we arrive at equations (29) and

$$
\begin{gathered}
\dot{\mathcal{K}}_{-}=\mathcal{K}_{-} A(t)+A^{\prime}(t) \mathcal{K}_{-}+\beta(t) \mathcal{K}_{-}+r(t)\left(\mathcal{K}_{-} S(t) \mathbf{C}(t)+\mathbf{C}(t) S^{\prime}(t) \mathcal{K}_{-}\right) \\
-\beta(t) G^{\prime}(t) R(t) G(t)=0, \mathcal{K}_{-}\left(t_{0}\right)=X^{0}
\end{gathered}
$$


Here $r=r(t)>0$ is a tuning parameter and

$$
\beta(t)=\alpha(t) /\left(1+\int_{t}^{\vartheta} \alpha(s) d s\right)
$$

THEOREM 5.3. The following inclusion (internal ellipsoidal estimate) holds

$$
\mathcal{X}[t] \supseteq \mathcal{X}_{-}[t]
$$

with

$$
\left.\mathcal{X}_{-}(t)\right)=\left\{x:\left(x-x^{*}(t), \mathcal{K}_{-}^{-1}(t)\left(x-x^{*}(t)\right) \leq 1\right\},\right.
$$

whatever be the orthogonal matrix $S(t)$ and function $0<r(t)<r_{0}$.

In order to check the property of being informative for measurement $y(t)$ one may use instead of $\mathcal{F}(s)$ the internal ellipsoidal approximations

$$
\left.G(t) \mathcal{E}_{-}\left(x^{*}(t), X_{-}[t]\right)\right) \subseteq G(t) \mathcal{G}\left(s, t, \mathcal{X}_{-}[t]\right)
$$

defined according to [24].

6. Conclusion. In this paper we introduced approximate estimates of solutions $V(t, x, z)$ to the HJB equations that arise in the problem of guaranteed (setmembership) filtering. These functions $V(t, x, z)$, which are the information states for this problem, allow one to describe the set-valued guaranteed estimates of the state vector - the information sets - on the basis of available observations (measurements). The information states are level sets $\mathcal{X}[t]$ of the information states. Upper and lower estimates for $V(t, x, z)$ thus allow one to calculate the external and internal set-valued estimates for $\mathcal{X}[t]$. In the case of linear systems these estimates turn out to belong to certain families of parameterized ellipsoids for which explicit equations are derived. A proper selection of the parameters (which is yet to be investigated) may allow one to achieve tight approximations or something close to these. The formulas derived here introduce one more new type of ellipsoidal solutions to the set-membership estimation problem (see also [25]).

\section{REFERENCES}

[1] R. Bellman and R. Kalaba, Dynamic Programming and Modern Control Theory. London math. society monographs, London, 1965.

[2] G. Leitmann, Optimality and reachability via feedback controls. In: Dynamic Systems and Mycrophysics, Blaquière A., Leitmann G., eds., 1982.

[3] D. P. Bertsekas, Dynamic Programming and Optimal Control. Athena Scientific, v.I,II, Belmont, Mass., 1995.

[4] A. B. Kurzhanski and P. Varaiya,Dynamic optimization for reachability problems. Journal of Optimization Theory and Applications, 108:2(2001), pp.227-251. 
[5] R. IsaAcs, Differential Games. John Wiley, New York, 1965.

[6] N. N. Krasovski and A. N. Subbotin, Game-theoretical Control Problems. Springer-Verlag, N.Y., Berlin, Heidelberg, 1988.

[7] T. Basar and P. Bernhard, $H^{\infty}$-Optimal Control and Related Minmax Design Problems. Second ed., Birkhäuser, 1995.

[8] A. B. Kurzhanski, Games of Observation, Soviet. Math. Doklady, 13:6(1972), pp.1556-1560.

[9] F. Schweppe, Uncertain Dynamic Systems, Prentice Hall, Englewood Cliffs, N.J., 1973.

[10] A. B. Kurzhanski, Control and Observation Under Uncertainty, Nauka, Moscow, 1977.

[11] F. L. Chernousko, State Estimation for Dynamic Systems, CRC Press, 1994.

[12] M. Milanese, et al (eds), Bounding Approach to System Identification, Plenum Press, 1995.

[13] A. B. KuRzhanski, On the problem of control synthesis under measurement feedback, PMM (Applied Mathematics and Mechanics), 68:4(2004), pp. 547-563.

[14] S. Osher and R. Fedkiw, Level Set Methods and Dynamic Implicit Surfaces. Springer. Ser. AMS., V.153, 2003.

[15] J. A. Sethian, Level Set Methods and Fast Marching Methods. Second.ed. Cambridge Univ. Press, 1999.

[16] A. Bensoussan and J-L. Lions, Contrôle Impulsionnel et Inequations Quasivariationelles. Bordas, Paris, 1962.

[17] F. H. Clarke, Yu. S. Ledyaev, R. J. Stern, and P. R. Wolenski, Nonsmooth Analysis and Control Theory. Springer-Verlag, Ser. GTM. V.178., 1998.

[18] V. I. Gurman, The Extension Principle in Problems of Control, Fizmatlit, Moscow, 1997.

[19] M. G. Crandall, L. C. Evans, and P-L. Lions, Some properties of viscosity solutions of Hamilton-Jacobi equations. Trans. Amer. Math. Soc., 282:2(1984), pp. 487-502.

[20] W. H. Fleming And H. M. Soner, Controlled Markov Processes and Viscosity Solutions. Springer-Verlag, N.Y., 1993.

[21] M. Bardi and I. Capuzzo-Dolcetta, Optimal Control and Viscosity Solutions of HamiltonJacobi-Bellman Equations. SCFA, Birkhäuser. Boston, 1997.

[22] A. I. Subbotin, Generalized Solutions of First-order PDE's. The Dynamical Optimization Perspective, SCFA, Birkhäuser. Boston, 1995.

[23] A. B. Kurzhanski And I. VÁlyi, Ellipsoidal Calculus for Estimation and Control, SCFA, 1997, Birkhäuser. Boston.

[24] A. B. Kurzhanski and P. Varaiya, Ellipsoidal Techniques for Reachability Analysis: Part I : External Approximations. Part II: Internal approximationa. Box-valued Constraints. In: Optimization. Methods and Software, Taylor and Francis, 2002, v.17, pp. 177-237.

[25] A. B. Kurzhanski And P. Varaiya, Ellipsoidal techniques for reachability under state constraints. SIAM Journal of Control and Optimization (To appear). 